\title{
An on-line VAD based on Multi-Normalisation Scoring (MNS) of observation likelihoods
}

\author{
Igor Odriozola, Inma Hernaez, Eva Navas \\ Aholab Signal Processing Laboratory, University of the Basque Country (UPV/EHU), \\ Bilbao 48016, Spain
}

\begin{abstract}
Voice Activity Detection (VAD) is an essential task in expert systems that rely on oral interfaces. The VAD module detects the presence of human speech and separates speech segments from silences and non-speech noises. The most popular current on-line VAD systems are based on adaptive parameters which seek to cope with varying channel and noise conditions. The main disadvantages of this approach are the need for some initialisation time to properly adjust the parameters to the incoming signal and uncertain performance in the case of poor estimation of the initial parameters. In this paper we propose a novel online VAD based only on previous training which does not introduce any delay. The technique is based on a strategy that we have called Multi-Normalisation Scoring (MNS). It consists of obtaining a vector of multiple observation likelihood scores from normalised mel-cepstral coefficients previously computed from different databases. A classifier is then used to label the incoming observation likelihood vector. Encouraging results have been obtained with a Multi-Layer Perceptron (MLP). This technique can generalise for unseen noise levels and types. A validation experiment with two current standard ITU-T VAD algorithms demonstrates the good performance of the method. Indeed, lower classification error rates are obtained for non-speech frames, while results for speech frames are similar.
\end{abstract}

Keywords: VAD, observation likelihood, MNS, on-line speech processing 


\section{Introduction}

Voice activity detection (VAD) is a very important part of expert systems based on speech interfaces. Using VAD, audio signals are split into autonomous speech segments before being passed to the subsequent modules. Two kinds of 5 errors must be considered: silence or noise segments being passed as speech (the non-speech error rate) and speech segments being misclassified as silences and then not being passed to the processing system (the speech error rate). Both must be kept low of course, but their importance depends on the needs and design of the expert system using the VAD.

VAD is typically the first module employed in acoustic processing systems. It is profusely used in the development of all kinds of expert systems. In Mporas et al. (2010) the authors use Automatic Speech Recognition (ASR) technology wwith a VAD to develop a dialogue system in a motorcycle environment. Principi et al. (2015) describe an integrated system for processing voice emergency com-

15 mands using a VAD followed by ASR. VAD and ASR technologies constitute the core of the speech interface in a system using a serious game to support therapy for mental disorders in Kostoulas et al. (2012). All these systems, based on ASR, require a very low ratio of lost speech frames in order for all the meaningful audio frames to be available to the recogniser. On the other hand, if non-speech segments are passed as speech the recogniser will still be able to detect them, as they typically have a silence (or non-speech) model. The main purpose of VAD in ASR interfaces is to eliminate long silences and split the audio stream into shorter, manageable segments. Additionally computation time is reduced and consequently so is the decoding response time.

ASR is not the only technology that requires a good VAD module. Tirumala et al. (2017) identify VAD as one of the research areas for speaker recognition. For instance, VAD is included in an intelligent porch system where people are identified by their voices before entering the house (Kuan et al., 2012). VADs are also an important module in speaker segmentation and clustering systems, so such as the diarisation system presented in Martínez-González et al. (2017). In 
addition, VAD is an essential module in expert systems that include emotion identification (Alonso et al., 2015). For speaker and emotion recognition systems the VAD employed requires a very low number of erroneously classified silence or noise frames, since silences or noise frames do not convey emotion or the 35 speaker's identity. A high non-speech error rate will thus lower the performance of the system. If however some speech frames are lost, the system will still be able to perform correctly.

Current VADs can be tuned to behave closer to one mode or to the other, though the ideal behaviour would of course be to reduce both non-speech and speech error rates as far as possible.

When the oral interface of an expert system picks up audio signals by means of different devices and in different environments, the VAD has to cope with different recording conditions, channel characteristics and noise levels. This is in fact the greatest challenge for the current ASR systems (Virtanen et al. 45 2012). VAD systems currently adapt different parameters to adjust to changing background noise conditions. However, this approach has its shortcomings: on the one hand, there is a need for an initialisation time over a segment to adjust the parameters, which introduces an undesirable delay. On the other hand, any incorrect estimation of the parameters will lead to uncertainty in the performance of the system (Graf et al., 2015). Training the VAD beforehand is one way to avoid the initial adaptation, but the trained system should be able to generalise to unseen channels or background noises. On-line VAD decision making is still a challenge.

From the point of view of acoustic features, very different parameters have been investigated: periodicity measure (Tucker, 1992, Hautamäki et al., 2007), zero-crossing rate (Benyassine, 1997), pitch (Chengalvarayan, 1999), Short Term Energy (STE) (Rabiner \& Sambur, 1975) and Long Term Energy (LTE) (Ghosh et al., 2011; Ma \& Nishihara, 2013), spectrum analysis (Woo et al., 2000, Marzinzik \& Kollmeier, 2002), cepstral distance (Pollak \& Sovka, 1995), Linear Pre60 dictive Coding (LPC) (Nemer et al. 2001) and combinations of different features 
(Tanyer \& Özer, 2000). More recent research has been focused on using multiple features to train a statistical model or classifier using machine learning techniques rather than on exploring more discriminative new acoustic features, which was the traditional trend.

Both Gaussian Mixture Models (GMM) and Hidden Markov Models (HMMs) have been tested in the context of VAD. In Tatarinov \& Pollák (2008), speech and non-speech segments are modelled by two HMMs. A simple grammar is used to model transitions from one HMM to the other and voice detection becomes a task of finding the best path through a recognition network. It is shown that a simple HMM-based VAD functions properly when clean signals are considered. In Kingsbury et al. (2002) the same HMM strategy is followed to deal with background noise, but acoustic features and normalisation operations are used along with the results conveyed by the HMMs. In Veisi \& Sameti (2012) several noisy HMMs are trained to detect different noisy non-speech segments.

75 In this paper we also use the approach of scores generated by the HMMs.

Varela et al. (2011) addresses the problem of far-field speaker interference in human-machine oral interaction. A decision tree (DT) is trained using the scores of speech/non-speech HMMs and additional information related to farfield speech. A Support Vector Machine (SVM) is used in Enqing et al. (2002) 80 to discriminate between speech and non-speech, and improved versions include Signal to Noise Ratio $(S N R)$ information as in Ramirez et al. (2006a b). Hybrid SVM/HMM architectures are also proposed for VAD in Tan et al. (2014) to retain the discriminative and non-linear properties of SVM while modelling the inter-frame correlation through a HMM. Results show a better performance for the SVM-based VAD system. However, relatively high speech error rates are still obtained. Our proposed VAD outperforms this technique and obtains a speech error rate more than three times lower.

More recently, neural networks (NN) have appeared in the literature of VAD approaches. For instance, Hughes \& Mierle (2013) uses a recurrent neural network (RNN) with perceptual linear prediction (PLP) features testing clean signals. Convolutional neural networks (CNN) are also used in Thomas et al. (2014) 
with mel-spectral coefficients, but adaptation with supervised data is needed for unseen channels. In Obuchi (2016) feature vectors consisting of log-mel filterbank energies are fed into a DT, an SVM and a CNN classifier. However, in this VAD approach several parameters must be adjusted to adapt to different noise conditions.

Regarding on-line performance, the current deep learning approaches tend to have very long inference times, mainly because neural network architectures are normally designed to be as complex as possible without considering real-time limitations (Sehgal \& Kehtarnavaz, 2018). An exception is the system introduced in Zhang \& Wu (2013), where a collection of different acoustic features are used to train a deep-belief neural network (DBNN). Extensive experimental results where different types of noise are tested show that it outperforms several reference VADs, even in real time. Nevertheless, this system has to compute almost 300 features in each frame, which increases system complexity. By contrast, our approach seems to get better results and is much simpler.

In this paper we present a simple but highly effective VAD based on a method that we have called Multi-Normalisation Scoring (MNS). This consists of classifying multiple observation likelihoods generated by an HMM trained with normalised Mel-Frequency Cepstral Coefficients (MFCC) corresponding to silence audio segments. Our proposed VAD technique makes use of a classifier which is trained beforehand, so that only a classification task needs to be performed when a new incoming speech frame arrives. This means that results are obtained on-line frame by frame and there is no need to adjust any parameter, so no initialisation period is needed. Furthermore, in comparison with two current standard ITU-T VAD algorithms, our VAD has proved to perform much better in labelling non-speech frames and to obtain similar results in labelling speech frames without increasing computing time. The VAD has been tested for different types of noise, as well as several $S N R$. The results show that our proposed ${ }_{120}$ VAD technique is able to generalise. However, noises not seen during training provoke a slight decrease in the results. 
Section 2 describes the general architecture of the VAD system proposed in this paper. Section 3 describes the MNS method and its motivation. Section 4 provides a short overview of the databases used. To assess the performance 125 of the new VAD, several databases have been chosen in an attempt to cover a variety of contexts and use a considerable amount of test speech material. The results of different experiments (under both clean and noisy conditions) are shown in Section 5 . Section 6 describes a validation experiment comparing the results with two standard VADs, and some conclusions are finally drawn in Section 7

\section{General architecture of the system}

The on-line VAD technique proposed in this paper consists of three core blocks, as shown in Fig. 1. The input to the system is a vector of MFCCs obtained from the current signal frame, and the output is a VAD label: speech or non-speech.

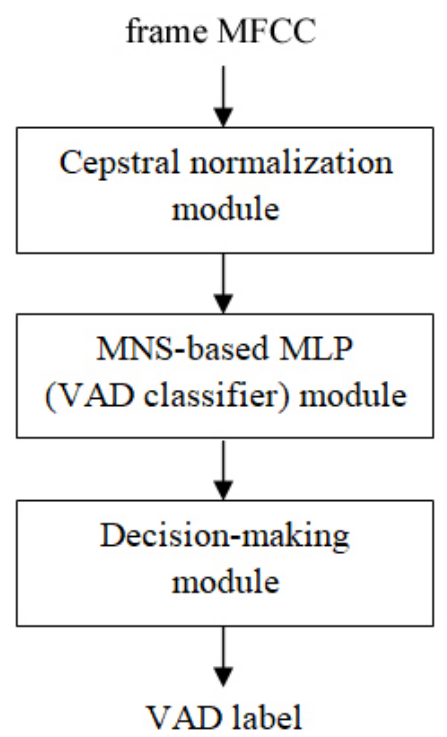

Figure 1: General architecture of the on-line VAD technique proposed here. 
The three core blocks are:

1. Cepstral normalisation module: the acoustic features (MFCCs) of the incoming signal frame are normalised using different normalisation factors.

2. MNS-based MLP (VAD classifier) module: this module classifies a vector obtained by our proposed Multi-Normalisation Scoring (MNS) method, using a Multi-Layer Perceptron (MLP).

3. Decision-making module: this implements a finite-state automaton to make immediate decisions in order to cope with glitches and enhance the results.

Block 2 implements the method presented here, and is described throughout the paper. Blocks 1 and 3 are described in more detail in the following subsections.

\subsection{Cepstral normalisation}

Cepstral normalisation is essential to develop the VAD proposed in this paper. Indeed, as demonstrated in earlier works (Westphal, 1997), the observation likelihoods generated by the silence GMM trained with normalised MFCCs follow a fairly discriminative pattern for speech and non-speech frames. The VAD proposed in this work takes advantage of this characteristic.

Overall, parameter normalisation is indispensable to create robust acoustic models and cope with audio signals captured in different environments. The spectral subtraction approach of Boll (1979) is well established in the ASR field for compensating for the differences (channels, background noise, etc.) in the incoming signals. However, the most common practice is to perform CMVN (Cepstral Mean and Variance Normalisation) on the extracted features, as it outperforms spectral subtraction techniques (Garner, 2011).

160

As explained in Huang et al. (2001), the mean of an MFCC over $N$ frames conveys the spectral characteristics of the current microphone and room acoustics. At the limit, when $N \longrightarrow \infty$ for each utterance, the means from utterances from the same recording environment can be expected to be the same. Thus, cepstral mean normalisation $(\mathrm{CMN})$ permits the removal of a stationary, linear 
The classic CMVN approach (Liu et al., 1993, 1994) seeks to estimate mean and variance vectors per cepstral feature (MFCC). The feature vectors are then shifted and scaled by the estimated means and variances, so that each normalised feature has zero mean and unit variance. An effective solution for calculating reliable means and variances is to estimate them using the whole utterance (offline performance). This utterance-based normalisation can result in undesirable delays, since utterance processing cannot begin until the last frame arrives. In time-synchronous (or on-line) systems, windows of a minimum length of 150means and variances and the latency. Once an initial value is estimated, some type of recursive normalisation is usually applied in which the long-term estimates for the means and variances of the cepstral features are incrementally updated.

The initial values for means and variances can be estimated using the first $M$ frames (and then adapting recursively). Correct estimation of these initial values depends heavily on whether these $M$ frames contain speech or not. If there is no speech in them, computed variance values will be very small, which will strongly amplify the amplitude of the normalised signal, and vice versa. In consequence, a good estimation of the initial values for means and variances is of the utmost importance. This issue can be overcome by using the method introduced in this paper, which is based on applying multiple normalisation factors to cepstral features. This enables decisions to be made frame by frame with no need to use a window.

\subsection{Decision-making module}

As the decision of speech/non-speech is made frame by frame, very short segments labelled as speech can appear in the output of the VAD. These short segments usually correspond to noises and glitches and degrade the performance of the following processing system. In an off-line implementation there is usually 
post-processing, but on-line implementation means making immediate decisions. In our on-line implementation, a classic state-diagram is implemented (see Fig. 2]. Two parameters are considered: minimum speech duration $\left(T_{\text {min_speech }}\right)$ and minimum silence duration $\left(T_{\text {min_sil }}\right)$, which set the minimum number of frames that a segment must contain to be considered as speech or silence (nonspeech), respectively. As can be seen in the figure, if the VAD changes its state from non-speech to speech (or vice versa) in a given frame, the next $T_{\text {min_speech }}$ frames (or $T_{\text {min_sil }}$ ) are also analysed. If the result of checking these frames matches the state of the current frame, a state change is made; otherwise it is assumed that there has been a glitch and the VAD does not change its state. Obviously, this method adds a short delay each time a state change is found, but no delay is added when the same state is maintained. This enables the system to completely recover during non-transitional segments.

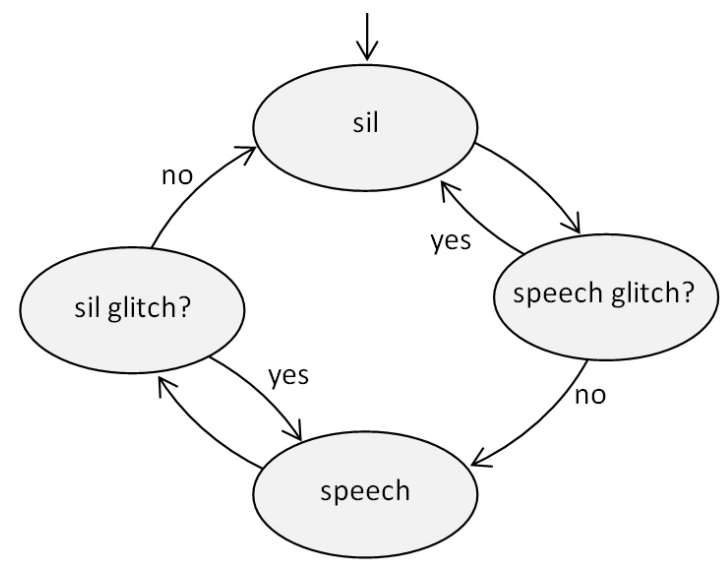

Figure 2: State-diagram for the on-line implementation of the decision-making module for glitch-removal.

For the experiments carried out in this paper, a minimum segment duration of 15 frames was empirically chosen for both $T_{\text {min_speech }}$ and $T_{\text {min_sil }}$. 


\section{The basis of the MNS method}

\subsection{Observation likelihood}

In speech recognition, audio segments corresponding to the same recognition unit (word, phone, triphone etc.) are gathered and processed in order to extract acoustic features (typically MFCCs) from them and train a different acoustic model for each unit. HMM is a very popular acoustic model, since it not only models the likelihood of a new observation vector but also the sequentiality of the observations.

Observation likelihoods are generated by GMMs, each of which corresponds to an HMM state. For an observation vector $o_{t}$, the observation likelihood $b_{j}$ of a GMM at the $j_{t h}$ state is calculated as shown in eq. 1

$$
b_{j}\left(o_{t}\right)=\sum_{m=1}^{M} c_{j m} N\left(o_{t} ; \mu_{j m}, \Sigma_{j m}\right)
$$

where $M$ is the number of mixture components, $c_{j m}$ is the weight of the $m^{t h}$ component and $N(\cdot ; \mu, \Sigma)$ is a multivariate Gaussian with mean vector $\mu$ and covariance matrix $\Sigma$.

\subsection{The observation likelihood of the central state of a three-state silence HMM}

The central state in a three-state HMM is a priori the most stable state of the model, since the left and right states have to cope with transitions between models. It makes sense to assume that the same goes for the silence HMM, where states at the ends have to model transitions between silence and speech.

An illustrative example is provided in Fig. 3, which shows the log-likelihoods generated by the GMM of each HMM state $\left(s_{0}, s_{1}\right.$ and $\left.s_{2}\right)$ through an utterance composed of three words (notice the mouth click just before the second word). The observation likelihood curve generated by the central-state $\left(s_{1}\right)$ GMM seems much more discriminative than the ones at the ends, which are more irregular. 

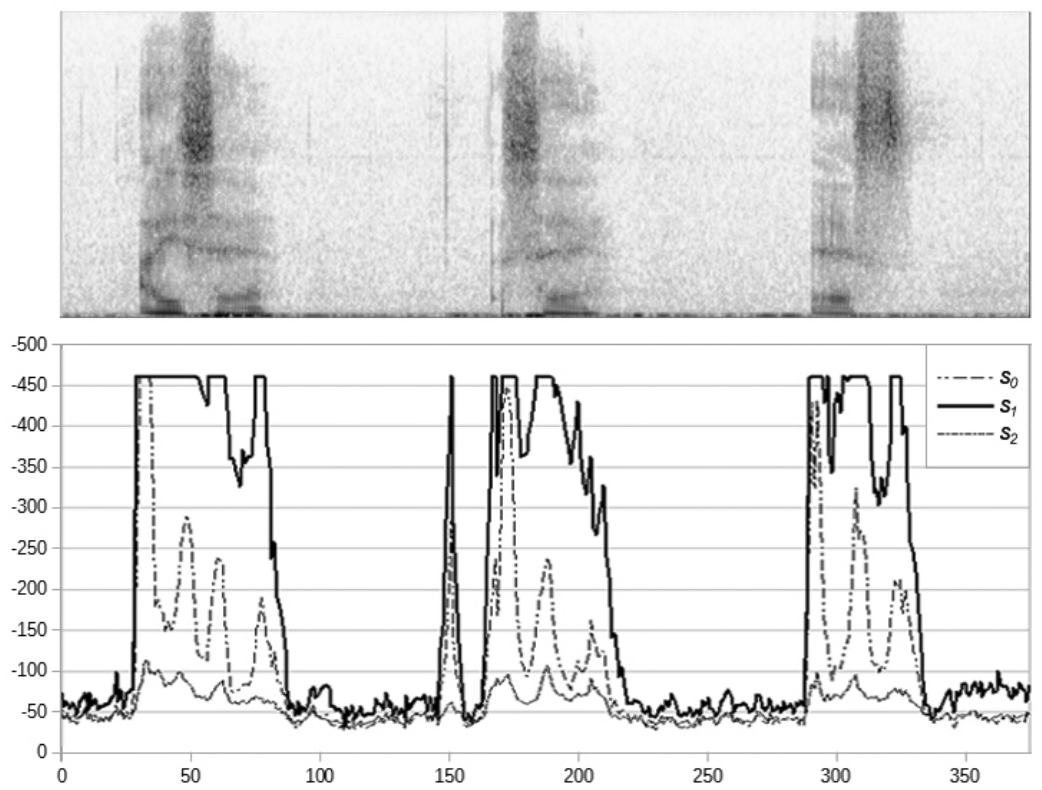

Figure 3: Spectrogram of an utterance consisting of three words (top) and observation loglikelihoods over time (frames) generated in the left state $\left(s_{0}\right)$, central state $\left(s_{1}\right)$ and right state $\left(s_{2}\right)$ of the silence HMM trained with normalised MFCCs (bottom).

\subsection{The Multi-Normalisation Scoring (MNS) method}

The MNS method consists of generating multiple observation likelihood scores by normalising the MFCCs using means and variances computed from different speech datasets obtained under different recording conditions. The observation likelihood vectors thus obtained can characterise the behaviour of the speech and non-speech frames in different conditions. As an illustrative example, Fig. 4 shows the behaviour of the scores obtained by normalising a signal picked up from near ( $B$ signal, top) and another from afar ( $E$ signal, bottom) with the pre-calculated means and variances obtained from four datasets recorded simultaneously at four distances: close $(B)$, desktop $(C)$, medium $(D)$ and far $(E)$ (for more details about the code names see Section 44).

Assuming that the differently normalised scores of the non-speech segments follow a pattern (see score vector $s_{i}$ in Fig. 4), it is likely that the speech scores 

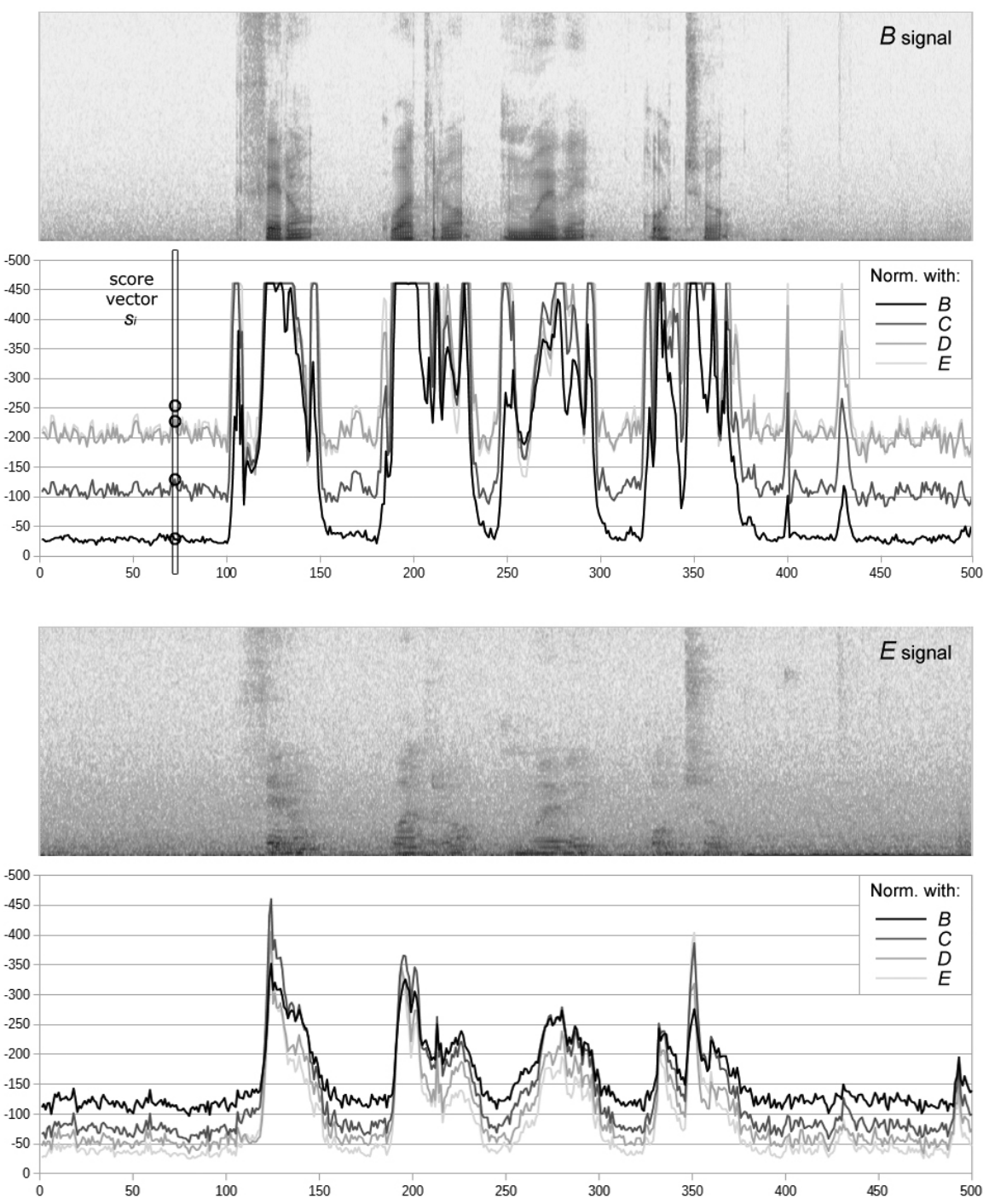

Figure 4: Spectrogram and central-state silence HMM observation log-likelihoods of a $B$ signal (top) and an $E$ signal (bottom) over time (frames) for different normalisation modes using pre-calculated means and variances from datasets $B, C, D$ and $E$. The vertical narrow box marks the vector of scores in frame $i$.

do likewise. If so, only a good classifier would be needed to detect those patterns and classify the vector as belonging to a speech or non-speech frame. 


\section{Speech databases}

250 Basque Speecon-like database (Odriozola et al., 2014), specifically the closetalk channel, to train the HMM for silence frames. Using this HMM, an MLP was trained by applying the MNS method to the files of the Basque Speeconlike database and a subset of the Spanish Speecon database used in an ECESS evaluation campaign of voice activity and voicing detection (Kotnik et al. 2008). The latter contains four channels or datasets corresponding to different recording distances: $C_{0}, C_{1}, C_{2}$ and $C_{3}$.

The initial VAD experiment was performed by testing the files from a third database: the TIMIT Acoustic-Phonetic Continuous Speech Corpus Garofolo 260 et al. 1993). The second VAD experiment was carried out by testing the system wwith noisy signals. For that purpose, the Noisy TIMIT speech database $\overline{\mathrm{Ab}}$ dulaziz \& Kepuska, 2017) was considered, in particular the babble noise dataset and white noise dataset Test blocks. Each dataset comprises 10 subsets each of which corresponds to a different $S N R$ (from 50 to $5 d B$, in $5 d B$ steps). For

the third VAD experiment, 4 of these 10 subsets $(35,25,15$ and $5 d B)$ were also included in the training material to train a new MLP, with the purpose of making the system more robust against noise. The files tested were the same as in the second experiment.

Finally, the results were compared using two standard VAD algorithms, and the same files as in experiments 2 and 3 were tested: the Test blocks of the babble noise and the white noise datasets of the Noisy TIMIT speech database.

Table 1 shows the main characteristics of the databases and the channels of each database used for this research. Each channel's code name as indicated in the table is used hereinafter.

\section{MNS-based VAD experiments}

The VAD accuracy experiments carried out in this study consist of assessing the ability of the system to discriminate between speech and non-speech 
Table 1: Main characteristics of the databases (and channels) used in this paper.

\begin{tabular}{|c|c|c|c|c|c|c|c|c|c|}
\hline Database & \multicolumn{2}{|c|}{$\begin{array}{l}\text { Basque Speecon- } \\
\text { like }\end{array}$} & \multicolumn{4}{|c|}{$\begin{array}{l}\text { Spanish Speecon - } \\
\text { ECESS }\end{array}$} & \multirow{2}{*}{$\begin{array}{l}\text { TIMIT } \\
F\end{array}$} & \multicolumn{2}{|c|}{ Noisy TIMIT } \\
\hline Code & $R$ & $A$ & $B$ & $C$ & $D$ & $E$ & & $G$ & $H$ \\
\hline Channels used & $\begin{array}{l}\text { Close- } \\
\text { talk }\end{array}$ & $\begin{array}{l}\text { Desktop } \\
\text { mic }\end{array}$ & $\begin{array}{l}\text { very } \\
\text { close } \\
\left(C_{0}\right)\end{array}$ & $\begin{array}{l}\text { close } \\
\left(C_{1}\right)\end{array}$ & $\begin{array}{l}\text { mediu } \\
\left(C_{2}\right)\end{array}$ & $\begin{array}{l}\text { afar } \\
\left(C_{3}\right)\end{array}$ & $\begin{array}{l}\text { Headset- } \\
\text { mounted and } \\
\text { far-field mic }\end{array}$ & $\begin{array}{l}\text { babble } \\
\text { noise } \\
(50-5 d B)\end{array}$ & $\begin{array}{l}\text { white } \\
\text { noise } \\
(50-5 d B)\end{array}$ \\
\hline Language & \multicolumn{2}{|l|}{ Basque } & \multicolumn{4}{|c|}{ Spanish } & English (USA) & \multicolumn{2}{|c|}{ English (USA) } \\
\hline Environment & \multicolumn{2}{|l|}{ Office } & \multicolumn{4}{|c|}{$\begin{array}{l}\text { Office, public place, } \\
\text { entertainment, car }\end{array}$} & Studio & \multicolumn{2}{|c|}{$\begin{array}{l}\text { Studio }+ \text { additive } \\
\text { noise }\end{array}$} \\
\hline Speakers & \multicolumn{2}{|l|}{230} & \multicolumn{4}{|l|}{60} & 630 & \multicolumn{2}{|l|}{630} \\
\hline Files / speaker & \multicolumn{2}{|l|}{316} & \multicolumn{4}{|l|}{17} & 10 & \multicolumn{2}{|l|}{600} \\
\hline Total content $(h)$ & \multicolumn{2}{|l|}{109.95} & \multicolumn{4}{|l|}{1.41} & 5.37 & \multicolumn{2}{|l|}{322.2} \\
\hline Speech content (\%) & \multicolumn{2}{|l|}{47.90} & \multicolumn{4}{|l|}{51.77} & 86.57 & \multicolumn{2}{|l|}{86.57} \\
\hline Labelling & \multicolumn{2}{|c|}{$\begin{array}{l}\text { Phonetic Forced } \\
\text { Alignment }\end{array}$} & \multicolumn{4}{|c|}{ Manually } & Manually & \multicolumn{2}{|c|}{ From TIMIT } \\
\hline Sample rate & \multicolumn{2}{|l|}{$16 \mathrm{kHz}$} & \multicolumn{4}{|c|}{$16 \mathrm{kHz}$} & $\begin{array}{l}20 \mathrm{kHz} \text { (down- } \\
\text { sampled } 16 \mathrm{kHz})\end{array}$ & \multicolumn{2}{|l|}{$16 \mathrm{kHz}$} \\
\hline
\end{tabular}

segments in terms of the non-speech error rate $\left(E R_{0}\right)$ and speech error rate $\left(E R_{1}\right)$. These two rates are computed as the fractions of the non-speech frames 280 and speech frames that are incorrectly classified $\left(N_{0,1}\right.$ and $N_{1,0}$, respectively) as a proportion of the number of real non-speech frames and speech frames in the whole database $\left(N_{0}^{r e f}\right.$ and $N_{1}^{r e f}$, respectively), as shown in equation 2 . In addition, the TER (total error rate) is also computed as the quotient between the total number of incorrectly classified frames and the total number of frames (equation 3).

$$
E R_{0}=\frac{N_{0,1}}{N_{0}^{r e f}} \times 100 ; E R_{1}=\frac{N_{1,0}}{N_{1}^{r e f}} \times 100
$$




$$
T E R=\frac{N_{0,1}+N_{1,0}}{N_{0}^{r e f}+N_{1}^{r e f}} \times 100
$$

The silence HMM was trained using the $R$ database. Acoustic parameters include 13 MFCCs and 13 first and 13 second order derivatives, and they were modelled with 32 mixture GMMs. The audio signals were windowed into 25 $m s$ length frames picking up a frame each $10 \mathrm{~ms}$. For the training of the silence HMM, these parameters were normalised using the means and variances computed from the files belonging to the same session (all the utterances corresponding to the same speaker).

Different classifiers were tested to see whether the scores obtained using the MNS method were valid, and the best results were obtained using a Multi-Layer Perceptron (MLP) (Widrow et al., 1988, Delashmit \& Manry, 2005), a classifier that can distinguish data that are not linearly separable (Collobert \& Bengio). For these experiments, MLPs were trained using WEKA (Waikato Environment for Knowledge Analysis), a popular free, open-source software written in the Java language for data-mining tasks (Holmes et al., 1994 Hall et al., 2009).

\subsection{MNS-based VAD experiment using an MLP}

To prepare the data to train the MLP, 1020 files of each of the datasets $R, A, B, C, D$ and $E$ were considered. All the files were processed to obtain observation likelihoods (generated by the central-state GMM of the three-state silence HMM trained with dataset $R$ ), after normalising the MFCCs using the means and variances precomputed from each dataset. Thus, vectors of 6 scores were generated from the frames of all the files belonging to each dataset. Altogether, 3096632 score vectors were obtained, $49.08 \%$ of which correspond to speech and $50.92 \%$ to non-speech, i.e. they are well balanced (for further details, see Table 3 .

The MLP used for this task contains 6 nodes in the input layer (one for each score) and 2 nodes in the output layer (one for each category: speech and 
non-speech). Half the sum of both node amounts (4 nodes) were chosen for the hidden layer.

To test the MNS-based MLP, a separate database was chosen: dataset $F$ 315 (TIMIT). All the files (6 300) from this dataset were processed in on-line mode; i.e. MFCCs were normalised on-line with the means and variances computed from subsets $R, A, B, C, D$ and $E$, giving a vector of 6 scores frame by frame. Then each score vector was classified by the MLP. The results of this experiment are shown in Table 2 ,

Table 2: $T E R, E R_{0}$ and $E R_{1}$ of the on-line VAD experiment on the TIMIT corpus.

\begin{tabular}{|l|l|l|}
\hline$T E R$ & $E R_{0}$ & $E R_{1}$ \\
\hline 4.98 & 19.68 & 2.70 \\
\hline
\end{tabular}

The results of the on-line MNS method proposed in this work can be considered as quite good when compared with other VADs, as shown in Section 6. $E R_{1}$ is low, which means that speech frames are quite correctly classified, so very few of them would be left out. However, $E R_{0}$ is quite high, with most of the errors being made at the ends of the speech segments. This means that almost one in five non-speech frames would pass on to the speech processing system.

\subsection{MNS-based VAD experiment in noisy conditions}

The MNS technique introduced in the previous section must be evaluated by testing noisy speech files in order to assess the robustness. For that purpose, two noisy datasets were considered: $G$ (babble noise) and $H$ (white noise), the most natural noises for a system hosted on a remote server. Both datasets contain 10 subsets with different $S N R$ s, ranging from 50 to $5 d B$ in $5 d B$ steps.

The experiment consisted of testing the same MLP used in the previous section (trained with clean signals) with the files corresponding to the Test blocks of datasets $G$ and $H$ (noisy signals): in total, 25200 files (1 260 files in 
each of the corresponding 20 different $S N R$ subsets). Fig. 5 shows the error rates obtained for both babble noise signals (orange dotted lines) and white noise signals (grey dotted lines) at different $S N R$ s. As a benchmark, the results presented in Table 2 when testing dataset $F$ (clean signals) are also shown in the figure, as horizontal black dotted lines.

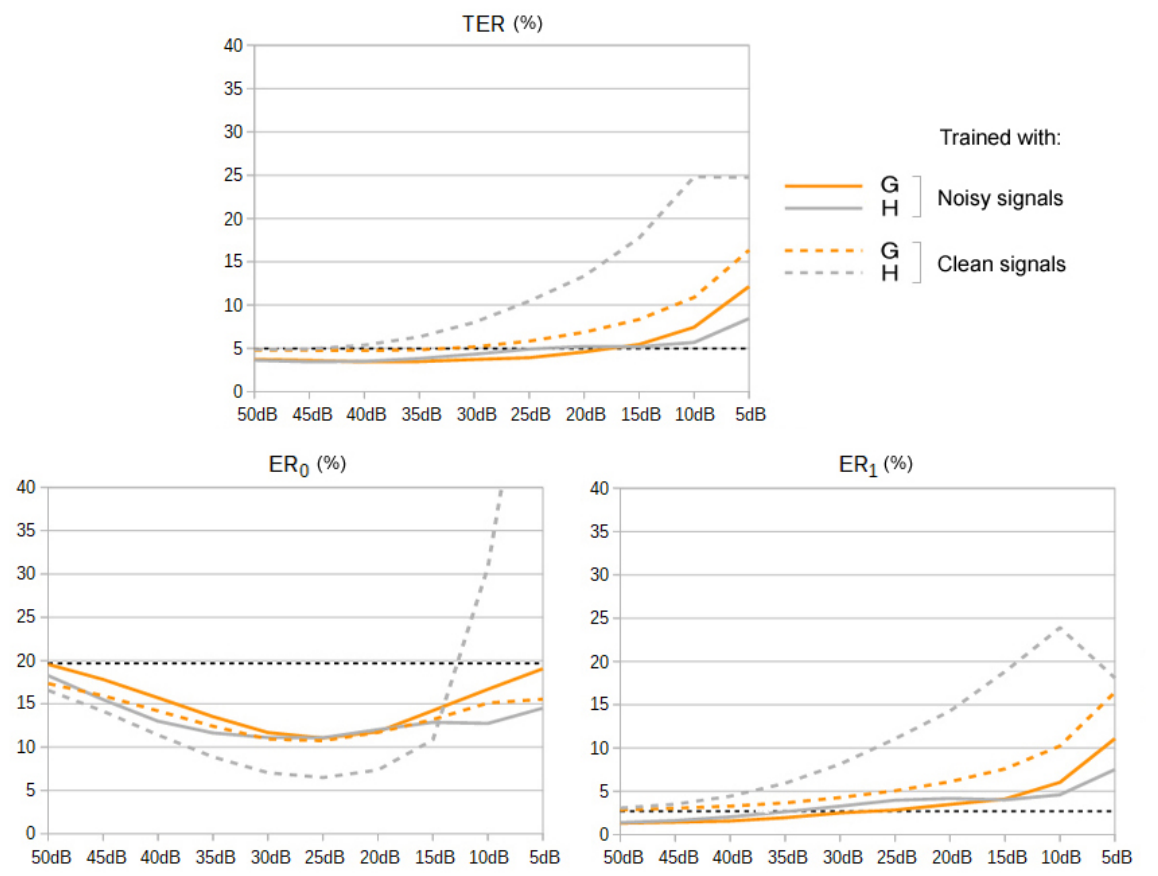

Figure 5: Error rates obtained by testing all the $S N R$ subsets of datasets $G$ and $H$ (noisy signals) when the MLP is trained using clean signals (dotted lines) and clean and noisy signals (solid lines); error rates obtained by testing clean signals are also shown as horizontal lines for reference (black dotted line).

The results show that testing noisy signals affects VAD performance: the $E R_{1}$ curve deteriorates in general, and the $E R_{0}$ results show a more irregular behaviour. Note the high $E R_{0}$ values obtained for white noise at the lowest $S N R$ s, probably due to the fact that white noise introduces energy at all frequencies and the MLP tends to classify all the frames as speech frames. Generally speaking, some deterioration was to be expected, since the scores used to train the MLP come from clean signals. That is why a new MLP was trained 
using scores obtained from noisy signals, as described in the next section. It should also be noted that the $T E R$ curve looks like the $E R_{1}$ curve, due to the fact that the $G$ and $H$ signals from the TIMIT database contain more speech $(86.57 \%)$ than non-speech (see Table 1).

\subsection{Training the MLP with noisy signals}

In this new experiment, noisy signals were included in the MLP training process. For this purpose, 4 subsets from each dataset $G$ and $H$ were chosen: the 35, 25, 15 and $5 d B$ (specifically, their Train blocks). Thus, the MLP training data now include the signals from the Train blocks of each of these 8 subsets, together with the files used in the MLP training process in the previous experiment. The results indicate whether the MLP is able to generalise when classifying signals with different $S N R$ s.

Since the TIMIT database is unbalanced in terms of the amount of speech and non-speech frames (see Table 1), a large number of speech frames were randomly discarded from the Train block files. Table 3 shows the total numbers of frames used per dataset.

Table 3: Datasets and numbers of frames considered to train the MLP with noisy signals.

\begin{tabular}{|l|c|c|c|c|}
\cline { 2 - 5 } \multicolumn{1}{c|}{} & $R, A$ & $B, C, D, E$ & $G, H$ & TOTAL \\
\hline non-speech fr & $299972 \times 2$ & $244211 \times 4$ & $190052 \times 8$ & 3097204 \\
speech fr & $234628 \times 2$ & $262647 \times 4$ & $244003 \times 8$ & 3471868 \\
\hline Total fr & $534600 \times 2$ & $506858 \times 4$ & $434055 \times 8$ & 6569072 \\
\hline
\end{tabular}

The score vectors now contain 14 elements: 6 scores obtained from the clean signals, and 4 from each of the subsets with noisy signals. Thus, the MLP configuration selected for this experiment is this: 14 nodes in the input layer, 2 nodes in the output layer, and 8 nodes for the hidden layer.

The files tested are the same ones as in the previous experiment (see Subsection 5.2 . Fig. 5 shows the error rates obtained at different $S N R$ s for both babble noise signals (orange solid lines) and white noise signals (grey solid lines). 
In the light of the results, there is an improvement in $E R_{1}$ at all levels of noise, for both dataset $G$ and dataset $H$, even when testing clean signals. Regarding $E R_{0}$, the improvement is remarkable for white noise at high noise levels. The big improvement in $E R_{1}$ together with the imbalance of the database 375 results in an overall improvement in TERs.

To show the impact of using noisy signals on the results obtained by testing clean signals, Table 4 presents the results of the experiment performed under clean conditions (see Table 2 along with the results obtained in this last experiment for the cleanest signals (50 dB subset). The results are actually even slightly better now.

Table 4: TER, $E R_{0}$ and $E R_{1}$ of the VAD experiment including noisy signals in the training process.

\begin{tabular}{|l|l|l|l|l|}
\cline { 3 - 5 } \multicolumn{2}{|c|}{ Exp. with clean signals } & $T E R$ & $E R_{0}$ & $E R_{1}$ \\
\hline \multirow{2}{*}{ Exp. with noisy signals } & babble noise $50 \mathrm{~dB}$ & 3.73 & 19.57 & 1.32 \\
\cline { 2 - 5 } & white noise $50 \mathrm{~dB}$ & 3.63 & 18.26 & 1.40 \\
\hline
\end{tabular}

\subsection{Generalisation to other types of noise}

We have shown that the MLP trained with 4 subsets (the 35, 25, 15 and 5 $d B$ ) of each dataset $G$ and $H$ is able to generalise results for the rest of $S N R$ values. However, seeking to learn whether the MLP trained with noisy signals can also generalise for other types of noises, we tested the MLP trained with noisy signals (see Subsection 5.3) with signals containing other types of noise. So now the test set comprises the files from the Test blocks of datasets $G$ (babble noise) and $H$ (white noise) along with the files belonging to the same Test blocks of the datasets blue, pink, red and violet (1260 files in each $S N R$ subset; 12600 in each dataset).

Figure 6 shows the $T E R \mathrm{~s}$ obtained at different $S N R \mathrm{~s}$ for the various noise types. For $S N R \mathrm{~s}$ equal to or greater than $35 d B$ there is no degradation when 
signals that have unseen noises are tested. For smaller $S N R \mathrm{~s}$, the deterioration is not very large: the maximum is for violet noise, which degrades by about 7 points at $15 d B$ with respect to both references. For red noise, the system actually behaves better than when the reference noises are tested.

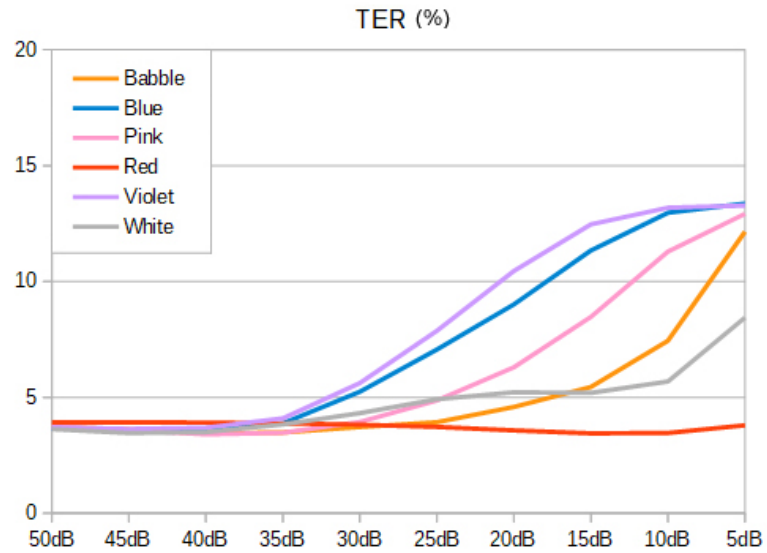

Figure 6: TERs obtained by testing all the $S N R$ subsets of all types of noise of the Noisy TIMIT, using the MLP trained with signals containing babble noise and white noise.

\section{Final experiments}

Two on-line VAD algorithms standardised by ITU-T (International Telecommunication Union - Telecommunication Standardization Sector) were tested to check the validity of the VAD technique proposed here. The algorithms belong to series G (Transmission systems and media, digital systems and networks), where G.710 - G.729 are devoted to Coding of voice and audio signals. The first algorithm is G.720.1 (1, ITU), which is actually a Generic Sound Activity Detector (GSAD) that can operate on 8 or $16 \mathrm{kHz}$ audio input, with a VAD module. The second algorithm is G.729 ([ITU), an $8 \mathrm{kbit} / \mathrm{s}$ speech coder that manages $8 \mathrm{kHz}$ input signals, which relies on a VAD module described in its Annex B (also known as G.729b). Both systems use a 10-ms frame length and frame shift, and no look-ahead is needed (no delay, just the frame duration). 
Further details are provided in Table 5 for both ITU system: $\rrbracket^{1}$ and our proposed VAD technique.

Note that the computation time is the average time per file needed by each system in a test where 10080 files are processed, using the same computer and under the same conditions. It can vary from one computer to another, but it gives some idea of the ratios between them. Additionally, regarding the hangover scheme, the G.729b and our proposed VAD technique follow a similar state machine, and introduce a delay while it is decided whether there is a change or not. In the case of G.720.1 a conservative scheme is followed, where active indicators are emitted until a silence segment is detected.

Table 5: Comparison of some important parameters of the VAD in G.720.1 (ITU-T), the G.729b algorithm (ITU-T) and our proposed VAD technique.

\begin{tabular}{|l|c|c|c|}
\cline { 2 - 4 } \multicolumn{1}{c|}{} & G.720.1 VAD & G.729b & Prop. method \\
\hline Bandwidth $(k H z)$ & 8,16 & 8 & 16 \\
Frame duration / shift $(m s)$ & $10 / 10$ & $10 / 10$ & $25 / 10$ \\
Computation time (ms per file) & 26.8 & 34.87 & 30.7 \\
Smoothing & No & Yes & Yes \\
Initialization (No. frames) & 200 inactive & 32 & 0 \\
\hline
\end{tabular}

To test the VADs, the same data were used as in Sections 5.2 and 5.3 . To 420 test the G.729 coder VAD, the files had to be down-sampled to $8 \mathrm{kHz}$. Fig. 7 shows the error rates obtained by the two ITU algorithms (dotted and dashed lines) and our proposed VAD technique (solid lines): $T E R$ (top), $E R_{0}$ (bottom left) and $E R_{1}$ (bottom right) testing the Test blocks of both datasets $G$ and $H$.

Regarding the $E R_{0}$, our proposed VAD technique lets at most $20 \%$ of nonspeech frames pass as speech. The minima of both ITU systems are over $30 \%$,

\footnotetext{
${ }^{1}$ The software for both systems can be downloaded from the ITU website: http: //www . itu . int/rec/T-REC-G.720.1-201001-I and http://www.itu.int/rec/T-REC-G.729-201206-I
} respectively. 

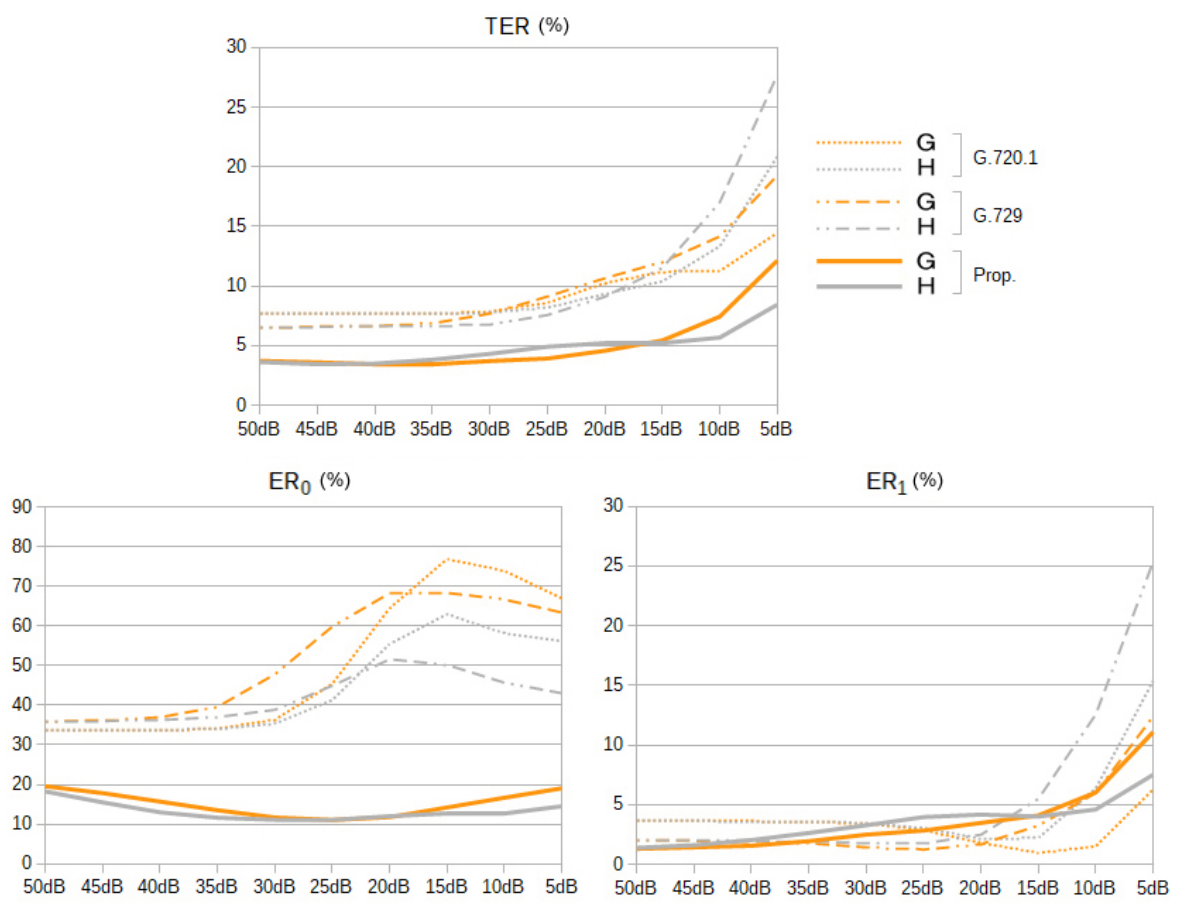

Figure 7: Error rates obtained using the ITU-T G.720.1 and G.729b standard VADs testing all the $S N R$ subsets of datasets $G$ and $H$, along with the results of the proposed VAD technique (solid lines).

and they show a significant increase as $S N R$ gets lower, especially for babble noise signals. This means that many non-speech frames are classified as speech when signals are noisy.

With regard to the $E R_{1}$, the results for G.720.1 and our proposed VAD technique are quite similar for babble noise. For white noise the results are similar for low-noise signals, but our proposed technique performs better on the noisiest data. In the case of G.729b, our proposed VAD obtains better results for $S N R$ higher than $25 d B$. At 10 and $5 d B$, G.729b gets better results for babble noise and the MNS-based VAD for white noise.

In general, $E R_{1}$ results obtained by the ITU algorithms and the MNS-based VAD are comparable for high $S N R$ signals. By contrast, for low $S N R$ signals 
the results show different behaviour for babble noise and white noise. For babble noise, G.720.1 gets similar results, and G.729b gets better results. For white noise, better results are obtained by the MNS-based system. Nevertheless, it is worth noting that $E R_{0}$ values are very high for the two ITU algorithms, which means that both systems tend to classify non-speech frames as speech when testing noisy signals.

In conclusion, our proposed VAD technique gets better TER at all noise levels. Due to the imbalance between the amount of speech and non-speech frames, the $T E R$ curves are similar in shape to those obtained for $E R_{1}$ but are shifted proportionally by $E R_{0}$. One of the advantages of the ITU systems is that they can adapt to different noise conditions on-line; however, they need an initialisation time to adjust the main parameters. In comparison, our MNSbased system is able to generalise for noise types that are not included in the training process and it requires no initialisation time, since the results do not depend on any previous frame.

\section{Conclusions and future work}

In this paper, we introduce a novel VAD that can be trained beforehand, so that it does not need any adaptive parameters or therefore any initialisation time to adjust those parameters. The VAD technique is based on the multi-normalisation scoring (MNS) method. MNS is based on generating an observation likelihood vector for each frame using the central-state GMM of a three-state silence HMM and normalising the cepstral features with different sets of means and variances. Thus, a classifier (a MLP) is trained using the vectors obtained from both speech frames and non-speech frames.

The performance of our proposed VAD technique when it is trained with noisy signals (babble noise and white noise) from different $S N R$ s is better overall than the performance of the ITU-T standard systems G.720.1 and G.729b, since the classification error is considerably lower for non-speech and is comparable for 
low speech error rates and systems that require low non-speech error rates. Furthermore, our VAD seems to generalise the results properly for intermediate $S N R$ s and the unseen noise types tested, which makes the system robust to different noise levels and types.

One of the greatest advantages of the MNS-based technique is that it performs on-line, making decisions frame by frame, with no need to analyse the neighbouring frames or the frames of a segment (or file) to which it belongs. In addition, the use of observation likelihoods as the basis of a VAD is also interesting due to its great simplicity. In a system where HMMs are used (as in an ASR system), the proposed VAD requires very little extra processing. The main disadvantage could be how the VAD behaves with unseen noises: it seems to be able to generalise results, but the error rate increases somewhat at some $S N R$ s. Further research is needed to determine how the system could perform a proper generalisation.

480

A future research direction could be the analysis of the observation likelihoods obtained from a speech GMM (or several GMMs). It would be interesting to see whether their incorporation deteriorates or improves the results. Acoustic models of speech are more diverse than those for silence, so the research should include the analysis of the various patterns obtained for different speech phones or phone groups. Additionally, several (noisy) silence GMMs trained with different noisy signals could also be considered. Indeed, all the work introduced here was carried out based on a single GMM trained with clean signals. Obtaining more score vectors from different silence GMMs might provide more stable results.

Further research is also needed to analyse the generalisation of results when processing audio signals containing unseen noises. Indeed, the ability to generalise is one of the keys of the proposed VAD technique, since it does not contain any adaptive parameters which can help to adjust the system to different noise types and levels. The impact of including different types of noise and different 495 combinations of them in the training data must be examined in depth to obtain a use that is as universal as possible. 
Another possible research direction could be to test different classifiers in addition to MLPs. Recurrent Neural Networks (RNN) seem to be a good candidate since they can model sequential data with time dependences between feature vectors. This might add robustness to the proposed MNS-based VAD technique.

A challenging research direction would be to use our VAD technique in the field of acoustic event detection. It would be interesting to see how our proposed VAD behaves in scenarios where not only speech but noises of other kinds are presented and must be detected. An in-depth analysis would be required to identify what adaptations the VAD system would need.

Finally, in regard to the most practical aspect, the system needs to be tested in a real expert system. It needs to be implemented in a real-world application where an assessment must be carried out. This would give clues as to the real performance of our proposed VAD technique.

\section{Acknowledgements}

This work was partially supported by the EU (ERDF) under grant TEC201567163-C2-1-R (RESTORE) (MINECO/ERDF, EU) and by the Basque Government under grant KK-2017/00043 (BerbaOla). The authors would like to thank

515 all the other members of the Aholab Signal Processing Laboratory for ongoing discussions and contributions to these topics.

\section{References}

Abdulaziz, A., \& Kepuska, V. (2017). Noisy TIMIT speech (ldc2017s04). URL: http://hdl.handle.net/11272/UFA9N

520 Alonso, J. B., Cabrera, J., Medina, M., \& Travieso, C. M. (2015). New approach in quantification of emotional intensity from the speech signal: emotional temperature. Expert Systems with Applications, 42, 9554-9564. 
Benyassine, A. (1997). ITU-T Recommendation G.729 Annex B: a silence compression scheme for use with G.729 optimized for V.70 digital simultaneous voice and data applications. IEEE Communications Magazine, 35, 64-73.

Boll, S. (1979). Suppression of acoustic noise in speech using spectral subtraction. IEEE Transactions on Acoustics, Speech, and Signal Processing, 27, $113-120$.

Chengalvarayan, R. (1999). Robust energy normalization using speech/nonspeech discriminator for german connected digit recognition. In Proc. of European Conference on Speech Communication and Technology (EUROSPEECH) (pp. 61-64).

Collobert, R., \& Bengio, S. (). Links between perceptrons, MLPs and SVMs. In International Conference on Machine Learning (ICML) (pp. 23-30).

535 Delashmit, W., \& Manry, M. (2005). Recent developments in multilayer perceptron neural networks. In Proceedings of the 7th Annual Memphis Area Engineering and Science Conference, MAESC. Memphis, USA.

Enqing, D., Guizhong, L., Yatong, Z., \& Yu, C. (2002). Voice activity detection based on short-time energy and noise spectrum adaptation. In Proc. of IEEE International Conference on Signal Processing (ICSP) (p. 464467). IEEE.

Garner, P. N. (2011). Cepstral normalisation and the signal to noise ratio spectrum in automatic speech recognition. Speech Communication, 53, 9911001.

Garofolo, J., Lamel, L., Fisher, W., Fiscus, J., \& Pallett, D. (1993). DARPA TIMIT acoustic-phonetic continous speech corpus CD-ROM. NIST speech disc 1-1.1. NASA STI/Recon Technical Report N, 93.

Ghosh, P., Tsiartas, A., \& Narayanan, S. (2011). Robust Voice Activity Detection using long-term signal variability. IEEE Transactions on Acoustics, Speech, and Signal Processing, 19, 600-613. 
Graf, S., Herbig, T., Buck, M., \& Schmidt, G. (2015). Features for voice activity detection: a comparative analysis. Journal on Audio, Speech and Music Processing (EURASIP), 2015, 91.

Hall, M., Frank, E., Holmes, G., Pfahringer, B., Reutemann, P., \& Witten, I. (2009). The WEKA data mining software: an update. ACM SIGKDD 555 explorations newsletter, 11, 10-18.

Hautamäki, V., Tuononen, M., Niemi-Laitinen, T., \& Fränti, P. (2007). Improving speaker verification by periodicity based Voice Activity Detection. In Proc. of the International Conference on Speech and Computer (SPECOM) (pp. 645-650).

560 Holmes, G., Donkin, A., \& Witten, I. (1994). Weka: a machine learning workbench. In Intelligent Information Systems,1994. Proceedings of the 1994 Second Australian and New Zealand Conference on (pp. 357-361).

Huang, X., Acero, A., \& Hon, H.-W. (2001). Spoken Language Processing: A Guide to Theory, Algorithm, and System Development. (1st ed.). Upper Saddle River, USA: Prentice Hall PTR.

Hughes, T., \& Mierle, K. (2013). Recurrent neural networks for voice activity detection. In Proc. of IEEE International Conference on Acoustics, Speech, and Signal Processing (ICASSP) (pp. 7378-7382).

(ITU), I. T. U. (2010). Recommendation ITU-T G.720.1: Generic Sound Activity Detector (Series G: Transmission Systems and Media, Digital Systems and Networks: Digital Terminal Equipments - Coding of Voice and Audio Signals). Technical Report Telecommunication standardization sector of ITU (ITU-T). URL: https://www.itu.int/rec/T-REC-G.720.1.

(ITU), I. T. U. (2012). Recommendation ITU-T G.729: Coding of speech at 8 575 kbit/s using conjugate structure algebraic-code-excited linear prediction (CSACELP) (Series G: Transmission Systems and Media, Digital Systems and Networks: Digital Terminal Equipments - Coding of Voice and Audio Signals). 
Technical Report Telecommunication standardization sector of ITU (ITU-T). URL: https://www.itu.int/rec/T-REC-G.729

Kingsbury, B., Saon, G., Mangu, L., Padmanabhan, M., \& Sarikaya, R. (2002).

Robust speech recognition in noisy environments: The $2001 \mathrm{ibm}$ spine evaluation system. In Proc. of IEEE International Conference on Acoustics, Speech, and Signal Processing (ICASSP) (pp. I-53-I-56).

Kostoulas, T., Mporas, I., Kocsis, O., Ganchev, T., Katsaounos, N., Santamaria,

J. J., Jimenez-Murcia, S., Fernandez-Aranda, F., \& Fakotakis, N. (2012). Affective speech interface in serious games for supporting therapy of mental disorders. Expert Systems with Applications, 39, 11072-11079.

Kotnik, B., Sendorek, P., Astrov, S., Koc, T., Ciloglu, T., Fernández, L. D., Banga, E. R., Höge, H., \& Kačič, Z. (2008). Evaluation of voice activity and voicing detection. In Proc. of Annual Conference of the International Speech Communication Association (INTERSPEECH) (pp. 1642-1645).

Kuan, T.-W., Tsai, H.-C., Wang, J.-F., Wang, J.-C., Chen, B.-W., \& Lin, Z.-Y. (2012). A new hybrid and dynamic fusion of multiple experts for intelligent porch system. Expert Systems with Applications, 39, 9288-9296.

Liu, F.-H., Stern, R., Acero, A., \& Moreno, P. (1994). Environment normalization for robust speech recognition using direct cepstral comparison. In Proc. of IEEE International Conference on Acoustics, Speech, and Signal Processing (ICASSP) (pp. II-61).

Liu, F.-H., Stern, R., Huang, X., \& Acero, A. (1993). Efficient cepstral normalization for robust speech recognition. In Proc. of ARPA workshop on Human Language Technology (HLT) (pp. 69-74).

Ma, Y., \& Nishihara, A. (2013). Efficient voice activity detection algorithm using long-term spectral flatness measure. Journal on Audio, Speech and Music Processing (EURASIP), 2013, 87. 
artínez-González, B., Pardo, J. M., Echeverry-Correa, J. D., \& San-Segundo, R. (2017). Spatial features selection for unsupervised speaker segmentation and clustering. Expert Systems with Applications, 73, 27-42.

Marzinzik, M., \& Kollmeier, B. (2002). Speech pause detection for noise spectrum estimation by tracking power envelope dynamics. IEEE Transactions on Speech and Audio Processing, 10, 109-118.

Mporas, I., Kocsis, O., Ganchev, T., \& Fakotakis, N. (2010). Robust speech interaction in motorcycle environment. Expert Systems with Applications, 37, $1827-1835$.

Nemer, E., Goubran, R., \& Mahmoud, S. (2001). Robust voice activity detection 615 using higher-order statistics in the LPC residual domain. IEEE Transactions on Speech and Audio Processing, 9, 217-231.

Obuchi, Y. (2016). Framewise speech-nonspeech classification by neural networks for voice activity detection with statistical noise suppression. In ICASSP (pp. 5715-5719).

${ }_{620}$ Odriozola, I., Hernaez, I., Torres, M., Rodriguez-Fuentes, L., Penagarikano, M., \& Navas, E. (2014). Basque speecon-like and basque speechdat mdb-600: speech databases for the development of asr technology for basque. In Proc. of the International Conference on Language Resources and Evaluation (LREC) (pp. 2658-2665).

625

Pollak, P., \& Sovka, P. (1995). Cepstral speech/pause detectors. In IEEE Workshop on Nonlinear Signal and Image Processing (pp. 388-391).

Principi, E., Squartini, S., Bonfigli, R., Ferroni, G., \& Piazza, F. (2015). An integrated system for voice command recognition and emergency detection based on audio signals. Expert Systems with Applications, 42, 5668-5683.

630

Rabiner, L., \& Sambur, M. (1975). An algorithm for determining the endpoints of isolated utterances. Bell Systems Technical Journal, 54, 297-315. 
Ramirez, J., Yelamos, P., Gorriz, J., Segura, J., \& Garcia, L. (2006a). Speech/non-speech discrimination combining advanced feature extraction and svm learning. In Proc. of Annual Conference of the International Speech Communication Association (INTERSPEECH) (pp. 1662-1665).

Ramirez, J., Yelamos, P., Gorriz, J. M., \& Segura, J. C. (2006b). SVM-based speech endpoint detection using contextual speech features. Electronic Letters, 42, 426-428.

Sehgal, A., \& Kehtarnavaz, N. (2018). A convolutional neural network smartphone app for real-time Voice Activity Detection. IEEE Access, 6, 9017-9026.

Tan, Y. W., Liu, W. J., Jiang, W., \& Zheng, H. (2014). Hybrid svm/hmm architectures for statistical model-based voice activity detection. In International Joint Conference on Neural Networks (pp. 2875-2878).

Tanyer, S., \& Özer, H. (2000). Voice activity detection in nonstationary noise. IEEE Transactions on Speech and Audio Processing, 8, 478-482.

Tatarinov, J., \& Pollák, P. (2008). HMM and EHMM based voice activity detectors and design of testing platform for VAD classification. Digital Technologies, 1, 1-4.

Thomas, S., Ganapathy, S., Saon, G., \& Soltau, H. (2014). Analyzing convolutional neural networks for speech activity detection in mismatched acoustic conditions. In Proc. of IEEE International Conference on Acoustics, Speech, and Signal Processing (ICASSP) (pp. 2519-2523).

Tirumala, S. S., Shahamiri, S. R., Garhwal, A. S., \& Wang, R. (2017). Speaker identification features extraction methods: A systematic review. Expert Systems with Applications, 90, 250-271.

Tucker, R. (1992). Voice activity detection using a periodicity measure. IEE Proceedings, Part I: Communications, Speech and Vision, 4, 377-380. 
Varela, Ó., Segundo, R. S., \& Hernández, L. A. (2011). Combining pulsebased features for rejecting far-field speech in a HMM-based Voice Activity Detector. Computers and Electrical Engineering, 37, 589-600.

Veisi, H., \& Sameti, H. (2012). Hidden-Markov-model-based voice activity detector with high speech detection rate for speech enhancement. IET Signal Processing, 6, 54-63.

Virtanen, T., Singh, R., \& Raj, B. (2012). Techniques for Noise Robustness in Automatic Speech Recognition. (1st ed.). Wiley Publishing.

Westphal, M. (1997). The use of cepstral means in conversational speech recognition. In Proc. of European Conference on Speech Communication and Technology (EUROSPEECH) (pp. 1143-1146).

Widrow, B., Winter, R., \& Baxter, R. (1988). Layered neural nets for pattern 670 recognition. IEEE Transactions on Acoustics, Speech, and Signal Processing, 36, 1109-1118.

Woo, K., Yang, T., Park, K., \& Lee, C. (2000). Robust voice activity detection algorithm for estimating noise spectrum. Electronic Letters, 36.

Zhang, X., \& Wu, J. (2013). Deep belief networks based Voice Activity De675 tection. IEEE Transactions on Acoustics, Speech, and Signal Processing, 21, $697-710$. 